\title{
Practical Advices About How to Handle Disease Progression During Osimertinib in EGFR-Mutant NSCLC Patients: Is it The Same Old Story?
}

Cortellini $A^{1,2 *}$, Buttitta $F^{3}$, Marchetti $A^{3}$, Ficorella $C^{1,2}$

${ }^{1}$ Medical Oncology, St. Salvatore Hospital, University of L'Aquila, L'Aquila, Italy

${ }^{2}$ Department of Biotechnological and Applied Clinical Sciences, University of L'Aquila, L'Aquila, Italy

${ }^{3}$ Center of Predictive Molecular Medicine, CeSI-MeT, University of Chieti-Pescara, Chieti, Italy

"Corresponding author: Dr. Alessio Cortellini, Medical Oncology Unit, St. Salvatore Hospital, Via Vetoio- 67100, L'Aquila, Italy, Tel: 00390862368709, Fax: 00390862368682; E-mail: alessiocortellini@gmail.com

Rec date: March 08, 2018; Acc date: March 24, 2018; Pub date: March 27, 2018

Copyright: (c) 2018 Cortellini A, et al. This is an open-access article distributed under the terms of the creative commons attribution license, which permits unrestricted use, distribution, and reproduction in any medium, provided the original author and source are credited.

\begin{abstract}
After the advent of third generation epidermal growth factor receptor (EGFR) tyrosine kinase inhibitors (TKls), oncologists are called to face new challenges in everyday management of EGFR mutated non-small-cell-lungcancer (NSCLC) patients. These drugs, in particular Osimertinib (which is the only one currently available), represent an extraordinary innovation. But while raising the bar of expectations, they pose us new challenges. Mechanisms of resistance to Osimertinib are heterogeneous: from a "molecular point of view" they can be categorized in EGFR-dependent and independent ones. In recent years many clinical reports have shown interesting results with target treatments, mainly chosen on the basis of the "molecular resistance". However, in common practice clinicians and patients must face off with the reality and with limited treatment options. It may be helpful to classify different clinical patterns of disease progression during treatment with Osimertinib. Treating a localized progression to a single organ certainly differs from treating a wide dissemination of disease, as well as treating symptomatic progressions differs from treating non-symptomatic ones. This mini-review aims to analyse, with a very practical approach, current options for clinical management of EGFR mutant NSCLC patient at the time of disease progression during Osimertinib, by focusing particularly on maintenance strategies beyond progression.
\end{abstract}

Keywords: Osimertinib; EGFR; NSCLC; Progression of disease; Mechanisms of resistance; Treatment beyond progression

\section{Introduction}

Lung cancer represents the most common oncological cause of death worldwide; non-Small-Cell-Lung-Cancers (NSCLCs) account for more than $80 \%$ of lung cancers and among them adenocarcinomas have become the most common histological type $[1,2]$. In NSCLC have been identified some "druggable" driver mutations and among them the most frequent and best-known ones are activating mutations of epidermal growth factor receptor (EGFR). They occur in about $10-15 \%$ of Caucasian patients with non-Squamous NSCLC [3] and they are predictive for response to EGFR tyrosine kinase inhibitors (TKIs). The latter are small molecules which inhibit the catalytic activity of the intracellular domain of the receptor. While in $E G F R$ wild type patients without other actionable biomarkers (such as $A L K$ translocations or PD-L1 overexpression) chemotherapy (platinum-based doublets or single agents, according to age, performance status and comorbidities) is still the best treatment choice, in EGFR mutant patients these compounds represent the standard of care [4]. The most common mutations of $E G F R$, which predict favourable response to first- and second-generation EGFR TKIs, are deletions in exon 19 and point mutations in exon 21 (mostly L858R) [5]. Other "rare" mutations have been identified and they are associated with de-novo resistance to firstand second-generation TKIs; among them exon 20 mutations (mostly T790M) are the best-known ones [6].

If we look at the availability of first- and second-generation EGFR TKIs (such as gefitinib, erlotinib and afatinib) in nowadays clinical practice, we can say "too much grace", because no one of them seems to overcome the others [7]. Thus, the decision-making process still follows a patient-by-patient algorithm, which is based on patient and disease' characteristics and on the expected toxicity profile. After the advent of third-generation EGFR TKIs, the clinical paradigm of these patients has radically changed. Since Clovis Inc. has interrupted the clinical development of Rociletinib in NSCLC [8], Osimertinib has become the only choice among third generation EGFR TKIs [9]; even considering other molecules which are in a much earlier phase of development [10].

The phase III study, that compared first line Osimertinib with firstgeneration EGFR TKIs in EGFR mutant advanced NSCLC patients, has shown advantageous preliminary results for Osimertinib [11]. Furthermore, FDA has granted a priority review to a supplemental new drug application to be used in this setting.

Nevertheless, current indication of Osimertinib is in patients who have progressed on a prior EGFR TKI treatment, with T790M EGFR mutation positive NSCLC $[9,12,13]$. Clinical management of these patients at the time of disease progression, after the "honeymoon" with the sequence of first EGFR TKI and Osimertinib, is now becoming a stumbling block which is hard to handle both for clinicians and patients. The aim of this mini-review is to find some firm points in this new scenario by taking into account previous experiences with firstand second-generation EGFR TKIs and by looking to the future perspectives. 


\section{Literature Review}

\section{Mechanisms of resistance and clinical patterns of progression}

Being a target therapy, clonal selection phenomena are inevitable. Thus, sooner or later, we have to reckon for every patient how to manage the disease progression. Different resistance mechanisms can arise during Osimertinib; in order to better understand them we can divide them into EGFR-dependent and independent ones.

Among EGFR a dependent mechanisms, there are mainly tertiary point mutations of exon 20; C797S is the best known one and it confers resistance to other third-generation inhibitors [14-16]. A typical feature of this kind of progression is that when C797S and the previous T790M are in trans position (i.e. in the different allele), tumour cells can retain sensitivity to the inhibition of EGFR axis by combining a first-generation TKI to a third generation one [17]. Other EGFR point mutations, described in plasma DNA, that confer resistance to Osimertinib are: L798I [18] and a case of solvent-front mutation at Gly796(G796S/R) combined with $\mathrm{C} 797 \mathrm{~S} / \mathrm{R}$ and $\mathrm{L} 792 \mathrm{~F} / \mathrm{H}$, all in cis position with previous T790M [19].

$E G F R$-independent resistance mechanisms are heterogeneous. First of all, we have to consider $E G F R$ wild-type amplification, which induce an over-activation of the axis [20] but also an aberrant downstream signal transduction can be involved. Activation of STAT3 and SrcYAP1 has been described to be able to confer intrinsic resistance to every kind of EGFR TKI [21], as well as HER2 and MET amplification and KRAS G12D point mutations [22-24]. A loss of EGFR T790M mutation was observed in addition to alternative pathways activation, including MPK1, AKT3, FGFR1 amplification and PTEN deletion [25]. In preclinical models a $B R A F$ V600E mutation, a $N R A S$ mutations and a gain of copy number of wild-type $N R A S$ and $K R A S$ have also been described [26,27]. Eventually even the transformation to small cell lung cancer must be taken into account [28].

The radiological finding of a dimensional increase of present lesions, the involvement of another organ or a new metastasis in the same organ, was historically accepted as the main criteria to discontinue an ongoing treatment. It is now clear that for a good management of disease progression, other clinical factors must be considered (diseaserelated and patient-related). RECIST and others evaluation criteria [29] are commonly accepted as standard guidelines for the evaluation of response. But sometimes, in the "real life", they can move oncologists away from the real goal of such type of treatments. In a metastatic disease, after two lines of therapy, the objectives of a disease-oriented treatment are to relieve symptoms and to improve quality of life, as well as prolonging survival. At the time of progression clinicians must have this concept clear in mind: it is crucial for an appropriate decision-making process and to properly weigh expected outcomes and risk/benefit ratio. For a systematic evaluation, before discussing the therapeutic choices, we have to categorize different patterns of disease progression.

From a "radiological point of view", among progressions (which have been defined according to commonly accepted evaluation criteria) we can distinguish between oligo-progressions and systemic progressions [30]. This differentiation between a localized progression to a single organ and a wide dissemination of disease, leads not only to clinical, biological and prognostic differences but also to different possible therapeutic approaches. In the first case adding a locoregional ablative therapy, mainly radiotherapy, by maintaining the same systemic treatment is a valid option (commonly used in clinical practice) $[31,32]$. In the second case a radical change of the systemic therapy is required.

A clinically useful differentiation is to distinguish between symptomatic and non-symptomatic disease progressions. With this in mind, a progression that leads to a worsening of disease-related symptoms necessarily require an integration to the ongoing therapy. Another differentiation can be made between life-threatening and non-life-threatening progression. In this case a progression to a "sanctuary site" (such as spinal cord, brain stem, or the involvement of a large artery), even without symptoms, obviously require a therapeutic adjustment.

\section{Discussion}

After the advent of target therapies, and even more of immune checkpoint inhibitors, it is becoming clearer and clearer: in case of indolent progression, maintaining the same treatment beyond disease progression is sometime the best choice to obtain a better quality of life and to prolong survival. Furthermore, these beyond progression strategies are also based on clinical trials [33].

Historically in the setting of disease progression to standard EGFR TKIs, among beyond progression strategies was included maintaining the EGFR TKI alone: continuously or after a "drug holiday" (in order to re-sensitize the molecular axis) in case of systemic indolent progression. Other "more interventional" options were: continuing the EGFR TKI adding chemotherapy (doublets or more frequently single agent) and in case of oligo-progression, particularly to the brain [34,35], continuing EGFR inhibition and adding a local-ablative therapy (such as radiation therapy). For a comprehensive analysis we have to keep in mind, not so much the lack of treatments options but, the poor effectiveness of these treatments in this kind of patients: they are often frail and with a worsening performance status at the time of disease progression. A recent "real-life" study in Caucasian EGFRmutated NSCLC patients, clearly demonstrated the abovementioned concept [36].

In the setting of disease progression during Osimertinib, after two lines of EGFR TKI therapy, the clinical management become harder to handle. Clonal selection could bring to a wide intra-tumoural heterogeneity, so the questions are: do we need a sniper or an infantry? Is it correct to look for a single druggable pathway, which explains alone the disease progression? Or should we look for a less (or not at all) clonal-targeted therapy such as chemotherapy? Or still, do immune checkpoint inhibitors represent a better choice?

There are two aspects to be evaluated for each patient: therapeutic alternatives and clinical pattern of progression.

Firstly, we have to admit that what we can do differs from what we could do; indeed, current possibilities after Osimertinib are limited. Some clinical experiences have reported interesting results with target treatments, which were selected on the basis of the identified molecular resistance pattern, such as re-challenges with firstgeneration EGFR TKI [17,37] or switch to MET inhibitors [23]. Another interesting possibility among adaptive therapies are the so called "drug holidays", which were already used in first- and secondgeneration EGFR TKIs. Indeed, this kind of strategy is theoretically applicable to all target therapy, in order to limit clonal selection [38]. However, in nowadays clinical practice there are just two choices: continuing Osimertinib beyond progression (always by evaluating the integration of a local therapy in case of oligo-progression) or switching 
to a chemotherapy regimen (a doublet when patient's clinical conditions allow it), or to an immune-checkpoint inhibitor. When considering treatment options, we have to face off with reality. Immune checkpoint inhibitors, which have radically changed current scenario of NSCLC, not seem to have done the same for EGFR mutant patients. A meta-analysis confirmed that immune checkpoint inhibitors do not improve OS over docetaxel in this population [39]. Since mutation burden showed to be a predictor of clinical benefit for these kind of treatments, the fact that EGFR mutant lung tumours have a low mutational load could be part of the explanation [40]. Regarding chemotherapy, there are neither prospective trials nor reported clinical experiences in the setting of progression to Osimertinib. On the other hand, considering that we have already discussed about the poor clinical outcomes achieved with chemotherapy at progression to firstgeneration EGFR TKIs, we are allowed to hypothesize that similar (or even worst) results are achievable even in this case. In our opinion, considering the favourable toxicity profile of Osimertinib (even more favourable if compared to chemotherapy) and the poor clinical conditions which often characterize this kind of patients, a maintenance strategy should always be taken into consideration. In case of indolent progression without symptoms or when an oligoprogression, even symptomatic, can be overcome with a local-ablative therapy, choosing a treatment with Osimertinib beyond progression can represent the best choice in current clinical practice. It is up to clinicians to discuss with patients and their families that it would not only be an exercise of patience, but also a practice of wisdom. In the unfavourable case of a wide spreading of disease, with symptoms which cannot be handled, albeit with all its limitations, it is mandatory to offer our patients a therapeutic alternative such as chemotherapy.

Following the logic of "the more you know the better you treat", a re-biopsy is always recommended at the moment of disease progression. However, in clinical practice, liquid biopsy is often a more convenient choice, probably because it is easier to perform in fragile patients thanks to its non-invasiveness. So, as clinicians have learned to use it for the routine diagnosis of T790M mutation at the time of disease progression during first line with standard EGFR TKIs [41], liquid biopsy can become a valid diagnostic tool even at this time of disease evolution. Furthermore, it can be a valuable support for monitoring treatment response and for the evaluation of resistances, as already shown in first line setting [42].

Future challenges in management of EGFR mutant NSCLC patients are about how to overcome emerging EGFR-dependent and independent forms of resistance. Many clinical trials are studying combination strategies of Osimertinib with other agents (such as immune checkpoint inhibitors, MET inhibitors, etc). Even if the most guideline-generating data will probably come from ongoing trials with third generation EGFR TKIs as first line treatment [10]. This strategy as well as a better control of disease, and in particular of central nervous system metastases, could delay the onset of molecular resistance [43].

\section{Conclusion}

As we previously said, the aim of this manuscript is not to speculate on future perspectives. Its purpose is to try to reflect, from a practical point of view, about how to improve clinical management of EGFR mutant NSCLC patients at the time of disease progression during Osimertinib in current clinical practice. In our opinion, considering the actual lack of available treatment options, a treatment with
Osimertinib beyond progression should always be taken into account, especially in case of indolent or oligo-progressions.

\section{Acknowledgements}

None.

\section{Conflicts of Interest}

The authors declare that they have no competing interests.

\section{References}

1. Jemal A, Bray F, Center MM (2011) Global cancer statistics. CA Cancer J Clin 2011: 61: 69-90.

2. Forman D, Bray F, Brewster DH (2013) Cancer Incidence in Five Continents. IARC Press, Lyon, France.

3. Paez JG, Janne PA, Lee JC (2004) Activating (sensitising) EGFR mutations are predictive for response to the EGFR TKIs. Science 304: 1497-1500.

4. Novello S, Barlesi F, Califano R (2016) Metastatic non-small-cell lung cancer: ESMO Clinical Practice Guidelines for diagnosis, treatment and follow-up. Ann Oncol 1:1-27.

5. Zhang YL, Yuan JQ, Wang KF (2016) The prevalence of EGFR mutation in patients with non-small cell lung cancer: A systematic review and meta-analysis. Oncotarget 7: 78985-78993.

6. Yasuda H, Kobayashi S, Costa DB (2012) EGFR exon 20 insertion mutations in non-small-cell lung cancer: preclinical data and clinical implications. Lancet Oncol 13: e23-e31.

7. Liang W, Wu X, Fang W (2014) Network meta-analysis of erlotinib, gefitinib, afatinib and icotinib in patients with advanced non-small-cell lung cancer harboring EGFR mutations. PLoS One 9: e85245.

8. 8.http://www.fda.gov/downloads/AdvisoryCommittees/ CommitteesMeetingMaterials/Drugs/

OncologicDrugsAdvisoryCommittee/UCM494782.pdf

9. Santarpia M, Liguori A, Karachaliou N (2017) Osimertinib in the treatment of non-small-cell lung cancer: design, development and place in therapy. Lung Cancer 8: 109-125.

10. Russo A, Franchina T, Ricciardi GR (2017) Third generation EGFR TKIs in EGFR-mutated NSCLC: Where are we now and where are we going. Crit Rev Oncol Hematol 117: 38-47.

11. Soria JC, Ohe Y, Vansteenkiste J (2018) Osimertinib in Untreated EGFRMutated Advanced Non-Small-Cell Lung Cancer. N Engl J Med 2378: 113-125.

12. Bertranou E, Bodnar C, Dansk V (2018) Cost-effectiveness of osimertinib in the UK for advanced EGFR-T790M non-small cell lung cancer. J Med Econ 21: 113-121.

13. Odogwu L, Mathieu L, Goldberg KB (2017) FDA Benefit-Risk Assessment of Osimertinib for the Treatment of Metastatic Non-Small Cell Lung Cancer Harboring Epidermal Growth Factor Receptor T790M Mutation. Oncologist 1:1-2.

14. Thress KS, Paweletz CP, Felip E (2015) Acquired EGFR C797S mutation mediates resistance to AZD9291 in non-small cell lung cancer harboring EGFR T790M. Nat Med 21: 560-562.

15. Wang S, Tsui ST, Liu C, Song Y, Liu D (2016) EGFR C797S mutation mediates resistance to third-generation inhibitors in T790M-positive non-small cell lung cancer. J Hematol Oncol 9: 59.

16. Song HN, Jung KS, Yoo KH (2016) Acquired C797S mutation upon treatment with a T790M-specific third-generation EGFR inhibitor (HM61713) in non-small cell lung cancer. J Thorac Oncol 11: e45-e47.

17. Niederst MJ, Hu H, Mulvey HE (2015) The allelic context of the C797S mutation acquired upon treatment with third-generation EGFR inhibitors impacts sensitivity to subsequent treatment strategies. Clin Cancer Res 21: 3924-3933. 
Citation: Cortellini A, Buttitta F, Marchetti A, Ficorella C (2018) Practical Advices About How to Handle Disease Progression During Osimertinib in EGFR-Mutant NSCLC Patients: Is it The Same Old Story?. J Mol Genet Med 12: 338. doi:10.4172/1747-0862.1000338

Page 4 of 4

18. Chabon JJ, Simmons AD, Lovejoy AF (2016) Circulating tumour DNA profiling reveals heterogeneity of EGFR inhibitor resistance mechanisms in lung cancer patients. Nat Commun 7: 11815.

19. Ou SI, Cui J, Schrock AB (2017) Emergence of novel and dominant acquired EGFR solvent-front mutations at Gly796 (G796S/R) together with $\mathrm{C} 797 \mathrm{~S} / \mathrm{R}$ and $\mathrm{L} 792 \mathrm{~F} / \mathrm{H}$ mutations in one EGFR (L858R/T790M) NSCLC patient who progressed on osimertinib. Lung Cancer 108: 228231.

20. Nukaga S, Yasuda H, Tsuchihara K (2017) Amplification of EGFR wildtype alleles in non-small cell lung cancer cells confers acquired resistance to mutation-selective EGFR tyrosine kinase inhibitors. Cancer Res 77: 2078-2089.

21. Chaib I, Karachaliou N, Pilotto S (2017) Co-activation of STAT3 and YES-associated protein 1 (YAP1) pathway in EGFR-mutant NSCLC. J Natl Cancer Inst. 109: 1-2.

22. Planchard D, Loriot Y, Andre F (2015) EGFR-independent mechanisms of acquired resistance to AZD9291 in EGFR T790M-positive NSCLC patients. Ann Oncol 26: 2073-2078.

23. Ou SH, Agarwal N, Ali SM (2016) High MET amplification level as a resistance mechanism to osimertinib (AZD9291) in a patient that symptomatically responded to crizotinib treatment post-osimertinib progression. Lung Cancer 98: 59-61.

24. Ortiz-Cuaran S, Scheffler M, Plenker D (2016) Heterogeneous mechanisms of primary and acquired resistance to third-generation EGFR inhibitors. Clin Cancer Res 22: 4837-4847.

25. Kim TM, Song A, Kim DW (2015) Mechanisms of Acquired Resistance to AZD9291: A Mutation-Selective, Irreversible EGFR Inhibitor. J Thorac Oncol 10: 1736-1744.

26. Eberlein CA, Stetson D, Markovets AA (2015) Acquired resistance to the mutant-selective EGFR inhibitor AZD9291 is associated with increased dependence on RAS signaling in preclinical models. Cancer Res 75: 2489-2500.

27. Ho CC, Liao WY, Lin CA (2017) Acquired BRAF V600E mutation as resistant mechanism after treatment with osimertinib. J Thorac Oncol 12 : 567-572.

28. Ham JS, Kim S, Kim HK (2016) Two cases of small cell lung cancer transformation from EGFR mutant adenocarcinoma during AZD9291 treatment. J Thorac Oncol 11: e1-e4.

29. Tirkes T, Hollar MA, Tann M (2013) Response criteria in oncologic imaging: review of traditional and new criteria. Radiographics 33: 1323-1341.
30. Hellman S, Weichselbaum RR (1995) Oligometastases. J Clin Oncol 13: 8-10.

31. Villaruz LC, Kubicek GJ, Socinski MA (2012) Management of non-small cell lung cancer with oligometastasis. Curr Oncol Rep 14: 333-341.

32. Rusthoven CG, Yeh N, Gaspar LE (2015) Radiation Therapy for Oligometastatic Non-Small Cell Lung Cancer: Theory and Practice. Cancer J 21: 404-412.

33. Yerushalmi R, Gelmon K (2010) Treatment beyond progression: Is it moving from Belief to Evidence? The Oncologist 15: 796-798.

34. Yap TA, Macklin-Doherty A, Popat S (2017) Continuing EGFR inhibition beyond progression in advanced non-small cell lung cancer. Eur J Cancer 70: 12-21.

35. Campo M, Al-Halabi H, Khandekar M (2016) Integration of Stereotactic Body Radiation Therapy with Tyrosine Kinase Inhibitors in Stage IV Oncogene-Driven Lung Cancer. Oncologist 21: 964-973.

36. Vavalà T, Follador A, Tiseo M, et al. (2016) Be-Positive: Beyond progression after tyrosine kinase inhibitor in EGFR- positive non-small cell lung cancer patients: Results from a multicenter Italian observational study. Lung Cancer 95:73-81.

37. Koyyala BVP, Batra U, Jain P (2017) Good response to erlotinib in a patient after progression on osimertinib: A rare case of spatiotemporal T790M heterogeneity in a patient with epidermal growth factor receptormutant nonsmall cell lung cancer. South Asian J Cancer 6: 179.

38. Amirouchene-Angelozzi N, Swanton C, Bardelli A (2017) Tumor Evolution as a Therapeutic Target. Cancer Discov1: 1-2.

39. Lee CK, Man J, Lord S (2017) Checkpoint Inhibitors in Metastatic EGFRMutated Non-Small Cell Lung Cancer-A Meta-Analysis. J Thorac Oncol 12: 403-407.

40. Spigel DR, Schrock AB, Fabrizio D (2016) Total mutation burden (TMB) in lung cancer (LC) and relationship with response to PD-1/PD-L1 targeted therapies. J Clin Oncol 34: 9017.

41. Chougule A, Basak S (2017) Epidermal growth factor receptor T790M testing in progressed lung cancer: A review of sensitive methods for analysis of tissue and liquid biopsy samples. Indian J Cancer 54: S45-S54.

42. Marchetti A, Palma JF, Felicioni L (2015) Early Prediction of Response to Tyrosine Kinase Inhibitors by Quantification of EGFR Mutations in Plasma of NSCLC Patients. J Thorac Oncol 10: 1437-1443.

43. Ramalingam SS, Ohe Y, Nogami N (2014) Pre-clinical and clinical evaluation of AZD9291, a mutation-specific inhibitor, in treatment-naïve EGFR mutated NSCLC. Ann Oncol 25: 14146-14164. 